\title{
Changing Pattern of Orthopaedic Trauma Admissions During COVID-19 Pandemic: Experience at a Tertiary Trauma Centre in India
}

\author{
M. S. Dhillon ${ }^{1} \cdot$ Deepak Kumar $^{1}{ }^{10} \cdot$ Uttam Chand Saini ${ }^{1} \cdot$ Himanshu Bhayana ${ }^{1} \cdot$ Nirmal Raj Gopinathan ${ }^{1}$. \\ Sameer Aggarwal ${ }^{1}$
}

Received: 3 July 2020 / Accepted: 17 August 2020 / Published online: 28 August 2020

(c) Indian Orthopaedics Association 2020

\begin{abstract}
Introduction COVID-19 has emerged as a medical threat to mankind, with a serious disruption of lifestyle in 2020. This has not only changed the way we live and work but has also changed the pattern of hospital admissions and medical care. To see if there was significant change in the pattern and management of trauma in our region, we evaluated data from our centre for the lockdown period and compared it with data from the previous year, and also with some available international data. Methods We collated data from our Tertiary care hospital for two periods, i.e. from 25th March 2020 to 3rd May 2020 signifying strict lockdown and then from 4th May to 31st May during which some conditional relaxations were given. This was compared to data from similar periods in 2019. We looked at patient demographics, fracture types, injury mechanisms, and even changes in treatment protocols.

Results Significant reductions in caseloads were noted; open injuries were less, road accidents were infrequent, but cases due to falls, especially children and the elderly were still seen, although slightly reduced. The plan to minimize operative interventions could not be fully implemented due to complex nature of trauma seen by us. Only one case of bilateral amputation turned out to be positive, with no infectious consequences to the treating staff.

Conclusions COVID-19 pandemic led to significant reductions in trauma caseload and change in injury patterns. Doctor responses and patient management needs significant alteration to prevent spread of disease.
\end{abstract}

Keywords COVID-19 · Orthopaedic emergency · Coronavirus · Trauma demographics

\section{Introduction}

The viral pandemic which was first reported in December 2019 as a cluster outbreak in Wuhan, China [1] has spread across various countries and continents. India officially entered global havoc on 30th January with its first registered

Deepak Kumar

drdeepaknegimt@gmail.com

M. S. Dhillon

drdhillon@gmail.com

Uttam Chand Saini

Doc.uttamsaini@gmail.com

Nirmal Raj Gopinathan

nirmalpgimsortho@ rediffmail.com

Sameer Aggarwal

drsameer35@yahoo.co.in

1 Department of Orthopedics, PGIMER, Chandigarh, India
COVID case. WHO called it a global health emergency and called for a combined international effort to suppress the outbreak [2]. Various countries have adopted aggressive mitigation and containment measures such as complete lockdown. The government of India also enforced a strict nationwide lockdown from 24th March, which was initially scheduled for 21 days (till 14th April 2020), which was later extended till 3rd May. Following this, the lockdown was extended for 28 days (4th May to 31st May) with some conditional relaxations. During this phase of extended lockdown, the districts were classified into three zones i.e. red, orange and green, and various relaxations were applied accordingly. Resumption of services in phased manner was declared from 1 st June which has been termed as "unlock 1".

Implementation of lockdown led to remarkable decline in public mobility, which was hypothesised, would affect the epidemiology and profile of trauma patients presenting to the orthopaedic department. We reviewed the demographic data of the patients presenting with orthopaedic injuries to our 
level 1 trauma center from 25th March to 31st May 2020, to evaluate the change in patterns of patients and presentation over the various phases of the lockdown; this was subsequently compared with data acquired in the same time frame in the previous year, 2019. This observational study aimed to document the epidemiological profile of patients, type of injuries sustained and common mode of injuries during lockdown phase; to analyse the change in number and pattern of injury from the previous year. The findings from the study could be used for formulation of protocol for management of patients as well as resources in future pandemics.

\section{Materials and Methods}

This was a retrospective observational study performed at a tertiary care referral centre in North India. Data were collected from the hospital records department and counter checked from daily hospital admission and discharge records available in emergency and wards. Patients presenting to the orthopaedic emergency with fresh injuries, or those that were referred for tertiary level management were included in the study. Patients who presented with injuries more than 2 weeks old, presenting for postoperative follow-ups and patients not requiring admission were excluded from the study. Variables such as demographic profile, nature of injury, mechanism of injury and working diagnosis were collected. Two periods were studied for 2020; the first 40 days of lockdown (25th March to 3rd May 2020) hereafter referred as Phase 1, and 4th May to 31st May 2020, hereafter referred to as Phase 2, where some relaxations had been allowed (Fig. 1). During the lockdown period in accordance with the institutional COVID committee, all patients requiring admission had to undergo COVID testing and depending upon the level of emergency and COVID result, patients were sent to designated areas as per the protocol (Fig. 2). Data for the corresponding time frames from 2019 (previous year) were taken as the baseline to evaluate the changes in patterns and management protocols. Departmental review board approved the study as it was purely a databased observational study, with no patient intervention. All the data were collected on Microsoft excel (version 2016) spreadsheet for analysis and Chi-square test was performed to determine the level significance. A $p$ value of $<0.5$ was considered statistically significant.

\section{Results}

In phase 1 of lockdown (25th March to 3rd May 2020), 100 patients ( 2.5 patients/day) were admitted, of which 79 were males and 21 were females (Table 1). The number of admissions per day increased to 5.8/day $(n=163)$ during Phase 2 of lockdown (4th May to 31st May), of which 135 were males and 28 were females. A total of nine paediatric patients (age $<12$ years) were admitted during phase $1(0.22$ admissions per day). The number of paediatric patients during phase 2 increased to 17 (0.6 admissions per day). During phase 1 , the number of elderly patients (age $>60$ years) admitted were 17 ( 0.42 per day), while the number of elderly admitted in phase 2 were 19 (0.67 per day). During phase 1 , the number of cases with open fractures was 0.9 per day, which increased to 2.3 per day in phase 2. During phase 1 , $76 \%$ of patients $(76 / 100)$ underwent surgical intervention, while $88 \%$ of patients (144/169) underwent surgical intervention during Phase 2.

Road traffic accidents (RTA) accounted for 40 admissions (1 per day) during phase 1 , while the number increased to
Fig. 1 Graphic demonstration of comparative data of Phase 12020 with similar period on 2019

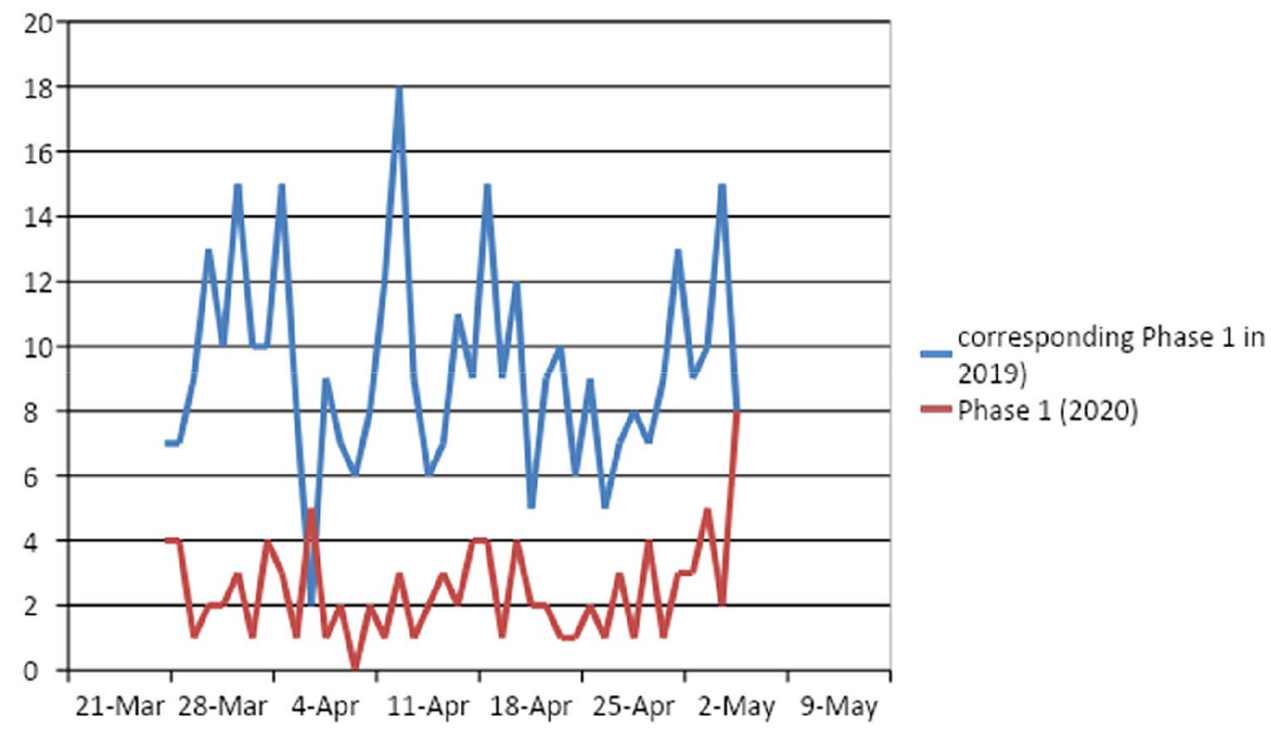


Fig. 2 Flowchart showing protocol adopted for initial management of trauma patients during lockdown phase

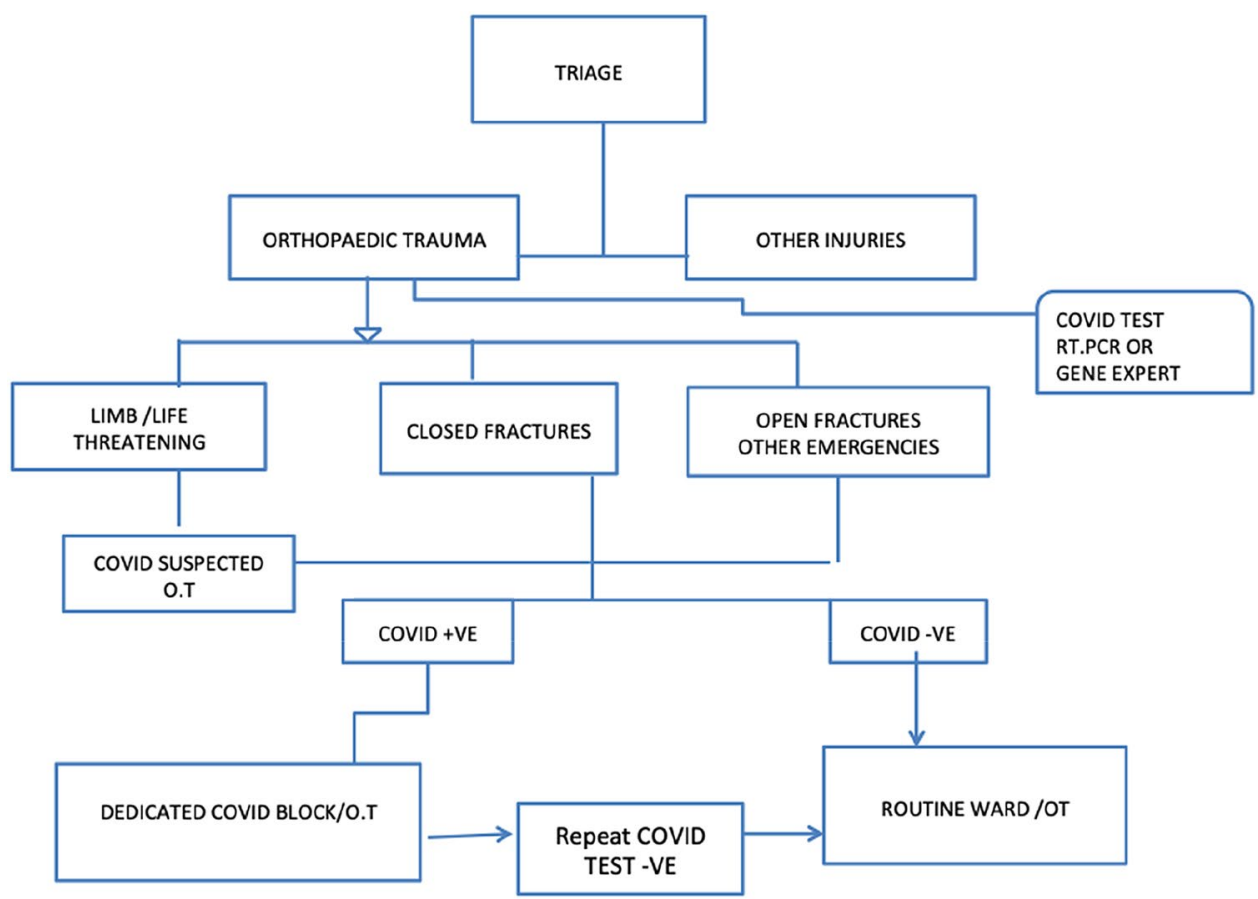

Table 1 Table depicting epidemiology of patients during the two phases of lockdown and in comparison to previous year

\begin{tabular}{lllll}
\hline & $\begin{array}{l}\text { Phase 1 2020 } \\
\text { 25th March-3rd May } \\
2020\end{array}$ & $\begin{array}{l}\text { Phase 2 2020 } \\
\text { 4th May-31st may 2020 } \\
(28 \text { days) }\end{array}$ & $\begin{array}{l}\text { 25th March-3rd May } \\
2019 \\
\text { (40 days) }\end{array}$ & $\begin{array}{l}\text { 4th May-31st may 2019 } \\
(28 \text { days) }\end{array}$ \\
& 100 & 163 & 364 & 247 \\
\hline Total number of patients & 2.5 & 5.8 & 9.1 & 8.8 \\
(No./day) & $79 / 21$ & $135 / 28$ & $311 / 53$ & $194 / 53$ \\
Males/females & $(79 \% / 21 \%)$ & $(82.8 \% / 17.1 \%)$ & $(85.4 \% / 14.6 \%)$ & $(78.5 \% / 21.5 \%)$ \\
& $9[9 \%]$ & $17[10 \%]$ & $25(6.87 \%)$ & $23(6.87 \%)$ \\
Children $\leq 12$ years & 0.22 & 0.6 & 0.62 & 0.82 \\
No. of children/day & $17[17 \%]$ & $19[11 \%]$ & $47(12.91 \%)$ & $34(13.8 \%)$ \\
Elderly $\geq 60$ years & 0.42 & 0.67 & 1.02 & 1.2 \\
No. of elderly/day & $36 / 64$ & $65 / 98$ & $148 / 216$ & $(40.7 \% / 59.3 \%)$ \\
Open/closed fractures & $36 \% / 64 \%$ & $39.8 \% / 60 \%$ & 3.7 & $99 / 148$ \\
& 0.9 & $144 / 15$ & & $218 / 25$ \\
Open fractures/day & & $88 \% / 9 \%$ & $329 / 22$ & $(88.3 \% / 10.1 \%$ \\
& $76 / 17$ & 2.3 & $(90.4 \% / 6.1 \%)$ & 3.5 \\
\hline
\end{tabular}

89 (3.17 per day) (Table 2). Trauma due to slip and fall accounted for 20 admissions ( 0.5 admissions per day) during phase 1 which increased to 25 ( 0.89 admissions per day). The number of cases due to machine cut injury was 0.2 per day, which increased to 0.39 per day. There were no railway track injuries in either phases of lockdown.

The data from same periods in 2019 reveal the number of patients admitted in the period corresponding to phase 1 (25th March to 3rd May 2019) was 364 (9.1 per day), while the number of admissions per day in 2019 corresponding to phase 2 (4th May to 31st May 2019) was 247 (8.8 per day). The paediatric patients admitted were 25 (0.62/day) and 23 (0.82/day), respectively. Elderly patients admitted in 2019 in the time corresponding to phase 1 and phase 2 were 17 (0.42/day) and 34 (1.2/day), respectively (Table 1$)$. The number of open fractures per day in 2019 was much higher at 3.7 (corresponding to phase 1) and 3.5 (corresponding to phase 2) (Table 2). $90.4 \%$ and $88.3 \%$ patients underwent surgical intervention in 2019 in a time frame corresponding to phase 1 and phase 2 , respectively ( $p=0.0002)$. 
Table 2 Table depicting modes of injury during the two phases of lockdown in comparison to previous year

\begin{tabular}{lllll}
\hline Mode of Injury & $\begin{array}{l}\text { Phase 1 2020 } \\
\text { 25th March-3rd May } \\
2020 \text { (40 days) }\end{array}$ & $\begin{array}{l}\text { Corresponding } \\
\text { period 2019 } \\
\text { 25th March-3rd } \\
\text { May 2019 } \\
(40 \text { days) }\end{array}$ & $\begin{array}{l}\text { Phase 2 2020 } \\
\text { 4th May-31st } \\
\text { may 2020 } \\
(28 \text { days) }\end{array}$ & $\begin{array}{l}\text { Correspond- } \\
\text { ing period } \\
2019 \\
\text { 4th May-31st } \\
\text { may 2019 } \\
\text { (28 days) }\end{array}$ \\
\hline RTA & & & & $180(72.9 \%)$ \\
(No./day) & $40[40 \%]$ & $277[76.1 \%]$ & $89(54 \%)$ & $(6.42)$ \\
Slip and fall & $(1)$ & $(6.92)$ & $(3.17)$ & $26(10.5 \%)$ \\
No./day & $20[20 \%]$ & $20[5.49 \%]$ & $25(15.3 \%)$ & $(0.92)$ \\
Fall from height & $(0.5)$ & $(0.5)$ & $(0.89)$ & $26(10.5 \%)$ \\
No./day & $25[25 \%]$ & $49[13.46 \%]$ & $30(18.4 \%)$ & $(0.92)$ \\
Machine cut & $(0.62)$ & $(1.22)$ & $(1.07)$ & $7(2.83 \%)$ \\
No./day & $8[8 \%]$ & $7[1.92 \%]$ & $11(6.7 \%)$ & $(0.25)$ \\
Assault & 0.2 & $(0.17)$ & 0.39 & $3(1.21 \%)$ \\
Blast and fire arm & $5[5 \%]$ & $5[1.37 \%]$ & $4(2.4 \%)$ & $1(0.40 \%)$ \\
Railway accident & 0 & $2[0.55 \%]$ & $1(0.6 \%)$ & $2(0.81 \%)$ \\
\hline
\end{tabular}

RTA accounted for 277 (6.92 cases/day) and 180 (6.42 cases/day) in 2019 in a time frame corresponding to phase 1 and phase 2, respectively. Trauma due to slip and fall accounted for 20 cases and 26 in 2019, whereas there were seven admissions due to machine cut injuries in both durations corresponding to lockdown. There were four cases due to railway track injuries in 2019 corresponding to the time frame of phase 1 lockdown while corresponding time to phase 2 in 2019 accounted for two cases.

There was a significant reduction in the number of patients presenting with trauma due to RTA $(p=0.00001)$, slip and fall $(p=0.00001)$ and proportion of patients undergoing operative intervention $(p=0.0002)$ in phase $1 \mathrm{com}-$ pared to the corresponding time in 2019.

\section{Discussion}

The Merriam-Webster dictionary defines "lockdown" as "security measure taken during an emergency to prevent people from leaving or entering a location" [3]. The Indian government was sufficiently proactive to initiate this "security measure" against the spread of Coronavirus from 24th March 2020. This led to a fear of overflow of the hospitals with patients, and shortage of healthcare facilities. Most hospitals, including ours, had plans in place where even orthopaedic surgeons would have to work in areas needing intensive care. As the lockdown continued, production and distribution of essential commodities had slowed down drastically and using up resources would deplete them further. The Government felt the economic pinch, and was left with the difficult task of fine-tuning the balance between a slowing down of the economy and dealing with the pandemic health crisis. Subsequently, following the pattern all over the world, there were various conditional relaxations in the application of lockdowns from 4th May 2020 onwards.

The purpose of this study was to evaluate any changes in the epidemiological profile of the trauma patients presenting to orthopaedic trauma center during various stages of the lockdown. We could discern almost a doubling of trauma patients once the strict phase of the first lockdown was over, with road accidents becoming more than three times the number as compared to lockdown 1 . This could not be compared statistically, as the number of days of the two periods was different. However, the number of cases was still lower than the corresponding periods of the previous year, which implied that the restraints put in place by the government were sufficient to reduce mishaps initially, as is evident by the significant reduction of road accident cases seen. In the subsequent phase of lockdown, although reduced as compared to the previous year, RTA remained a major cause of trauma admissions; this implied that sufficient traffic was plying despite government guidelines in both phases of the lockdown. One fact of note, which may not be evidenced by statistics, was the reduction in actual numbers of open injuries in lockdown phase 1 (0.9/day as compared to 3.7/ day in 2019), reflecting again on population mobility and effectiveness of restrictions in the initial phase.

On specifically evaluating the change in incidence of trauma due to fall, a reduction in overall cases was noted, although as a percentage of cases presenting it was higher in both phases especially in phase $1(p<0.05)$. Paediatric trauma also followed similar trend as trauma due to fall but it was not statistically significant. For the elderly, the pattern was the same, but in both the age groups, this was not statistically significant, implying that falls of children and elderly continued to occur, despite people not venturing out of their homes. 
One fact of note was that the insignificant reduction in percentage of cases with open fractures and complex injuries, implying that whatever complex cases that presented to other centres during this period, were still being referred to our hospital.

As per the guidelines of various orthopaedic societies [4], an effort was made to reduce operative intervention where possible. Our data showed that although operative interventions were reduced to some extent, the change was not statistically significant, reflecting again upon the complex nature of injuries, which our institute deals with, which mostly require operative interventions.

A survey of international surgeons [5] revealed significant changes in trauma management and orthopaedic practice worldwide; 63 orthopaedic centres from 28 countries informed that $91 \%$ centres had a reduced workload and only $17 \%$ of these were doing elective surgeries. $30 \%$ of these had needed to deploy orthopaedic personnel to help in non-orthopaedic areas prior to May 15, 2020. This is similar to our experience, as our functioning has also drastically changed, and our personnel have been made ready since 1 st June 2020 to be used in high intensity areas if needed.

Lockdowns of varying degree were imposed in various countries worldwide, and our understanding is that these were not as strict as they were in India. We compared our preliminary observational data to studies evaluating orthopaedic patients in Spain [6] and New Zealand [7]. Although the studies were different from ours, data from Spain [6] noted a similar reduction in traffic and workplace accidents, with reductions in hospital admissions, but the number of geriatric hip fractures remained almost the same; this was somewhat different from our experience. Since our hospital also had a block designated as a COVID hospital, it is our impression that many elderly cases who would have come to our centre with hip fractures etc. were diverted to private hospitals due to the fear of acquiring the disease in our centre.

The New Zealand study [7] looked at variations of volume and pattern in a level 1 trauma centre over two 14-day periods; they noted a $43 \%$ reduction in injury related admissions, and $48 \%$ reduction in paediatric admissions, and documented a predominance of injury at home, specifically falls in all age groups. We also found overall reduction in number of paediatric and elderly patients but there was no significant difference in terms of percentage.

Under pandemic conditions, where resource utilisation needs to be optimal, it becomes important for orthopaedic surgeons to understand the epidemiological pattern of the patients who present to the trauma centres; this allows them to be better prepared and use the available resources wisely. Non-operative management protocols maybe used in some cases as per the recommendation of national bodies [8], but with care as we do not want trauma patients to come back with problems like malunion and non-union at a later date.

In Italy [7], hospital reorganisation was done in some areas, where patients not requiring multi-specialty care were shifted to two specific hubs created for orthopaedic injuries alone. All elective surgeries were stopped, as were routine outpatient services, and only emergency OPD services were functional; all patients in emergency were subjected to oropharyngeal swabs and kept in a separate room till tests determined their status. Subsequent segregation was done according to the reports. By the second phase of the lockdown, this was also the protocol being followed in our hospital.

One fear prevalent in the medical community was contact with COVID-19 patients and adaptations of interventions and protocols. In the total period analysed, we had only one case of bilateral lower limb amputation who subsequently became COVID positive; this lead to the patient being shifted to the COVID isolation centre for subsequent care; the team identified to have dealt with the case was put into isolation, and the whole area including operation room used for surgery were sterilised. Luckily, none of them tested positive, and our changed protocols in place are preventing this episode from being repeated.

Joob and Wiwanitkit [9] have documented a case of wrist fracture they operated in Thailand after he returned from Japan; this was in the early period of the pandemic and awareness levels were low. Hence, it becomes important for all trauma cases to be tested prior to interventions, unless these are life or limb threatening. Even in these cases, immediate tests should be sent, and surgery should proceed with all COVID precautions and that too in specified isolation areas with designated operation rooms. This has also been documented by Mi et al. [10] who came in contact with the initial cases in China, and this can serve as an eye-opener for all of us.

Our study has a few limitations, first its a retrospective study and data was collected from hospital records. Second, being based on hospital admissions, it might not be representative of the overall pattern of orthopaedic trauma; being a designated COVID hospital some people might have opted to visit other hospitals for treatment for cases that were not so severe, thus altering our data. The Government of India is currently allowing more freedoms, and the government has further eased the restrictions. The increase in the number of trauma cases continues, and it is expected to rise further owing to increased human interactions and traffic. The pandemic scenario is expected to stay with us for quite some time, and our experience with trauma admissions and alteration of standard management protocols that we have followed, may provide administrators and surgeons information about what to expect in a crisis like this, so that we are better prepared for future circumstances. However due to limitations of our data, and the fact that we had an evolving 
learning experience as the pandemic also evolved, does not allow us to give more specific recommendations beyond sharing our experiences.

Acknowledgement We thank Dr. Anurag Rana, Dr. Aditya Wardhan and Dr. Aditya Aggarwal for their help on data collation.

\section{Compliance with Ethical Standards}

Conflict of interest The authors declare that they have no conflict of interest.

Ethical standard statement This article does not contain any studies with human or animal subjects performed by the any of the authors.

Informed consent For this type of study informed consent is not required.

\section{References}

1. Zhu, N., Zhang, D., Wang, W., Li, X., Yang, B., Song, J., et al. (2020). A novel coronavirus from patients with pneumonia in China, 2019. New England Journal of Medicine, 382(8), 727-733.

2. WHO (2020) Coronavirus disease 2019 (COVID-19) situation reports. https://www.who.int/emergencies/diseases/novelcoron avirus-2019/situationreports. Cited Jun 20, 2020.

3. Merriamwebster.com [Internet] https://www.merriamwebster. com/dictionary/lockdown?utm_campaign=sd\&utm_mediu $\mathrm{m}=$ serp\&utm_source $=$ jsonld. Cited Jun 28, 2020.
4. Chhabra, H. S., Bagaraia, V., Keny, S., et al. (2020). COVID19: current knowledge and best practices for orthopaedic surgeons. Indian Journal of Orthopaedics, 54(4), 1-15. https://doi. org/10.1007/s43465-020-00135-1.

5. Lezak, B. A., Cole, P. A., Jr., Schroder, L. K., \& Cole, P. A. (2020). Global experience of orthopaedic trauma surgeons facing COVID-19: a survey highlighting the global orthopaedic response. International Orthopaedics, 44(8), 1519-1529. https ://doi.org/10.1007/s00264-020-04644-4.

6. Nuñez, J. H., Sallent, A., Lakhani, K., et al. (2020). Impact of the COVID-19 pandemic on an emergency traumatology service: experience at a tertiary trauma centre in Spain. Injury, 51(7), 1414-1418.

7. Zagra, L., Faraldi, M., Pregliasco, F., et al. (2020). Changes of clinical activities in an orthopaedic institute in North Italy during the spread of COVID-19 pandemic: a seven-week observational analysis. International Orthopaedics, 44, 1-8.

8. COVID-19 guidelines Indian Orthopaedic Association [internet]. https://www.ioaindia.org/COVID-19IOAguidelines.pdf. Cited 25 Mar 2020

9. Job, B., \& Wiwanitkit, V. (2020). Carpal fracture and COVID-19 infection: observation from Thailand. Indian Journal of Orthopaedics, 54(3), 393.

10. Mi, B., Chen, L., Xiong, Y., Xue, H., Zhou, W., \& Liu, G. (2020). Characteristics and early prognosis of COVID-19 infection in fracture patients. Journal of Bone and Joint Surgery, 102(9), $750-758$.

Publisher's Note Springer Nature remains neutral with regard to jurisdictional claims in published maps and institutional affiliations. 